\title{
The Resilience of Analog Tools in Creative Work Practices: A Case Study of LEGO Future Lab's Team in Billund
}

\author{
Nanna Borum, Eva Petersson Brooks, and Søren R. Frimodt-Møller \\ Department of Architecture, Design and Media Technology \\ Aalborg University Esbjerg, Denmark \\ $\{\mathrm{nb}, \mathrm{ep}, \mathrm{sfm}\} @$ create. aau.dk
}

\begin{abstract}
This paper discusses the use of digital and analog tools, respectively, in a creative industry. The research was done within the EU-funded research project IdeaGarden, which explores digital platforms for creative collaboration. The findings in a case study of LEGO ${ }^{\circledR}$ Future Lab, one of LEGO Group's largest innovation departments, show a preference for analog tools over digital in the creative process. This points towards a general need for tangible tools in the creative work process, a need that has consequences for the development of new digital tools for creative collaboration.
\end{abstract}

Keywords: digital tools, collaboration technology, creative work practices.

\section{Introduction}

This paper discusses to which extent work practices in the creative industries to day have gone digital, and to which extent they still rely on analog tools.

The research in this paper is a product of the EU-funded, international research project IdeaGarden, comprised of institutions from Austria, Germany, Switzerland, Greece and Denmark, and exploring the possibilities of digital platforms designated for creative collaboration. The main focus of IdeaGarden is collaborations on visual or tangible designs, because these collaborations highlight the need for other digital tools than those currently available for collaborating on text-based information (e.g. Google Docs, Evernote etc.) IdeaGarden includes three case studies that have been chosen in order to exemplify different environments in which such creative processes can take place: EOOS, a small Viennese design company, Muthesius Academy of Fine Arts and Design in Kiel, Germany, and finally, LEGO® Future Lab, which is, in comparison, a fairly large work unit that collaborates on generating new concepts for LEGO products, and due to the size of the unit has to put this creative process into very clear (infra)structures in order to keep the work of individual team members integrated in one big process. The present paper focuses on the LEGO Future Lab case study, because it shows a very clear tendency regarding the use of analog tools vs. digital, given that LEGO Future Lab is a work unit one would expect to be even more dependent on digitalized workflows than the other two cases, due to its size and international scope. 
In the first sections of the paper, the method and set-up of the case study are described. After this, the work processes that are practically limited to either analog or digital tools are discussed first. After this discussion, the paper addresses the different areas of LEGO Future Lab's workflow that include both digital and analog tools. Finally, the paper concludes with a short discussion of what the interplay between digital and analog tools in the creative working environment means for the development and success of new technologies that facilitate creative work.

\section{Related Work}

The notion of the 'paperless office', i.e. a workplace that is not dependent on any kind of analog tool, was first coined in 1980 by XEROX PARC, but in fact, the basic thought can be traced back to the mid 1800's when the telegraph was invented [1]. Sellen and Harper explain that through the years the 'paperless office' has been a goal for many companies as it is regarded to support a more efficient workflow, decrease costs and in addition improve the ecological profile of the company. Still there is a resilience of going from analog, in other words, paper documents to digital documents. Gaver [2] argues that the tangibility of paper affords different interactions that digital documents do. Especially, Gaver highlights that paper supports both formal and informal situations and, in addition, the format of the notes works as a memory-trigger regarding the level of formality of the situation for the designer afterwards. Similarly, Brereton and McGarry [3] also point to how physical objects function as an episodic memory trigger.

Other qualities are that paper is considered free of boundaries and that it is transformable in a different way than a regular text file.

For physical object in general e.g. Geyer \& Reiterer [4] point to the importance of spatial representation of artifacts, in order to facilitate collaborative design.

Some researchers have conceptualized design as a reflective conversation with physical materials in the situation [5], but more recent work has drawn attention to the way design artifacts can be catalysts for the (further) design process [6], [7], [3], as well as have an impact on the design situation in virtue of their material qualities [8]. Dorta et al. [7] argue that it is simply a necessity for designers to exteriorize their mental models, not only as physical but also sometimes as digital representations in order to engage in a discussion of these models. This process of exteriorization furthermore needs to be on going, i.e. reiterated as models develop, and be accessible for the designers wherever they work, such that design decisions can be revisited and reviewed.

In addition, due to their tangibility, physical objects simply afford interpersonal communication in a different way than digital ones, and therefore make different types of idea generation possible. Some studies on the use of physical models in the field of engineering do, however, suggest that a design process might stagnate, once a physical model of a design is built, given that only few changes are made to a model, once its physical representation is introduced [9]. 


\section{Case Description and Research Questions}

LEGO® Future Lab is a recent fusion between LEGO Concept Lab and LEGO New Business Group. LEGO Future Lab works with frontend innovation, more specifically, development of new toy product lines and concepts in close correspondence with the market.

This study focuses on the approximately 30 persons large office in Billund but also addresses how this office collaborates with the other international branches of the same department.

Already before the case study was carried out, a tendency presented itself in conversations with the creative practitioners at the three test beds involved in IdeaGarden, especially the practitioners from LEGO Future Lab: The relatively high dependence on analog tools throughout the creative process, in spite of the ready availability of digital tools at many stages of the creative process as well as for communication between co-workers. This is of particular interest when looking at LEGO Future Lab, given that LEGO Future Lab is a work unit one would, as hinted at earlier, expect to be even more dependent on digitalized workflows than the other two test beds, due to its size and international scope.

Consequently, the main research question that has guided the questions for the interviews at LEGO Future Lab is related to this tension between the dependence on digital tools on one hand, and the individual team member's preference for analog tools on the other. This question splits into the sub questions of how analog and digital tools, respectively, are being used in parts of the work processes. In the sections that follow, the documented interplay between analog and digital tools has been grouped into aspects related to (in random order) sharing information with others, note-taking, collaboration, communication with external collaborators, tools for presentation and working with physical models.

When the interviews were carried out, LEGO Future Lab was divided into three "streams", namely Insights and Early Innovation, Pipeline, and Launch. (Today, the two latter streams have been merged into one, but the different functions and roles the respective streams took up in the work process described in the following, are still carried out by different people.) The distribution of tasks were, in slightly simplified form as follows:

The Insights and Early Innovation reacts to a "brief" from a selection of LEGO's stakeholders invited to a so-called "gate meeting". A brief can be a request to develop something that will address a particular market or target group, or make use of a particular technology. Insights and Early Innovation then undertakes field research on the possible market, target groups or technologies in question. This research can involve interviews with target groups, presenting the group with early mock-ups and loosely formulated suggestions for play concepts, or simply document typical patterns of playing in a particular group. From the insights gathered via field research, the stream comes up with ideas for directions the new products could take. The design directions from the Insights and Early Innovation stream are handed over to the Pipeline stream, which develops a number of actual product ideas based on these directions. The selected stakeholders choose one or more of the product ideas to be 
developed for a product launch, a process carried out by the Launch stream. In addition, the Launch stream tests the almost-finished products together with children, working closely together with the market in order to get the packaging and model designs as fitting as possible.

All three streams work with both marketing and design, as the way the product is shaped is dependent on its possible market and vice versa. The IdeaGarden partners from Aalborg University Esbjerg interviewed two employees from each of the three streams - for each stream, one person who worked with design, and one person who worked with marketing or project management.

\section{Method}

In order to investigate the work practices at LEGO® Future Lab, five semi-structured situated interview sessions [10] were conducted over a three-day period. In total six participants were interviewed, the individual interviews lasting between 60-96 minutes (mean= 77,8 min). The sessions were carried out with an ethnographic approach (Pink, 2007) [11] putting an emphasis on observing the participants' work practices in their working environment. The interviews were audio recorded, and combined with photos and field notes, the data is detailed, thick and hence applicable for a case study analysis [12]. In the analysis process the interviews were transcribed and main themes were identified [13] and elaborately described.

The unit of analysis focused on five topics: (a) digital and analog tools in relation to specific work practices, (b) tools for internal collaboration, (c) tools for remote and external communication, (d) tools for presentations, and (e) working with physical models. The results of the case study shows that even though the creative team strives to keep their workflow digital, they still rely heavily on analog, paper-based tools and physical models, as well as regular face-to-face communication.

\section{$5 \quad$ Results}

\subsection{Digital and Analog Tools in LEGO® Future Lab}

The following sections will discuss digital and analog tools in relation to specific work practices of LEGO® Future Lab. Certain work practices are, however, almost entirely carried out using one specific group of tools, digital for some practices, analog for others.

The processes that are practically only carried out in the digital realm include different means of sharing larger or more formal pieces of information with other team members. Interviews indicate that file-sharing via LEGO Group's internal servers is the preferred option of the administrative personnel, while many of the creative workers prefer sending attachments via e-mail:

"I prefer the folder system, because outdated files fly around through e-mails and dead links rule. The problem with the folder system is that I read $60-70 \%$ of my mails 
on the phone, and from there I can not access the system so often, so it is actually nice that people attach the file, but it is problematical when people start to work on attached files since it results in multiple different copies." Michael, Project Leader of new products, Launch.

"[Speaking of the problem with multiple versions and the difficulties arising from files getting misplaced:] the designers have a bit of a mess going on. [...] it gets a bit confusing, or actually not confusing, but tiring." Sidd, Creative Lead, Launch.

Another aspect of the working process, which is primarily digital, is exploration of existing ideas or technological and design tendencies, which a member of Launch explained is typically done via the Internet, e.g. by looking at trending sites. If a team member stumbles on anything of interest he or she usually shares the information with the other team members via e-mail. LEGO Future Lab is currently developing an online platform where the whole of LEGO Future Lab can share information on inspirational trends, technologies and toys. In parallel, a similar platform is being developed, that allows digital access to several inspirational resources such as methodological tool kits and guidelines previously developed and utilized in connection with idea generation and design cycles in LEGO Future Lab. Until now these resources have mainly been stored in tangible, primarily paper and cardboardbased forms. Some resources are available in the shared office space in the form of e.g. a board with a set of guidelines. Each guideline, presented on a $15 \mathrm{~cm} \times 15 \mathrm{~cm}$ piece of thick cardboard, are velcroed to the board, enabling a co-worker to remove the tile temporarily and bring the information to their table for inspiration. Other resources such as a pocketsize Moleskin archive containing sets of guidelines have been distributed to all co-workers to bring with them when working off-location.

Also on the analog end of the digital-analog spectrum, one finds the personal notes taken by the individual team member. For this purpose, practically everyone uses post-its or other paper-based media:

"I use post-its. I do not like digital notes. Physical notes 'scream for attention' when they are present. It helps me remember, and I can add on to them, delete tasks and so on as the day goes by. I can easily sort the post-its by relevance and by urgency [...]

[Speaking of idea development:] In general, I sketch! If I need to get a hold on things, I take notes and try to find new connections. I could also partner up with a colleague and storyboard on the theme." Maiken, Digital Product Designer, Pipeline.

"I prefer to have things in print. Let us just say that I am not the one who saves the rainforest. I print a lot. I cannot really take it in if it is on a screen, I need to be able to write directly on things, and doodle and so on. [...] Also, I do not misplace it when I have it physically, and I remember things easier." Mette, Insights Manager, Insights and Early Innovation.

Only one interviewee expressed preference for digital note taking: "I use this [tablet] for personal notes because it is easy to carry around and you can quickly share the notes afterwards." Michael, Project Leader of new products, Launch. 


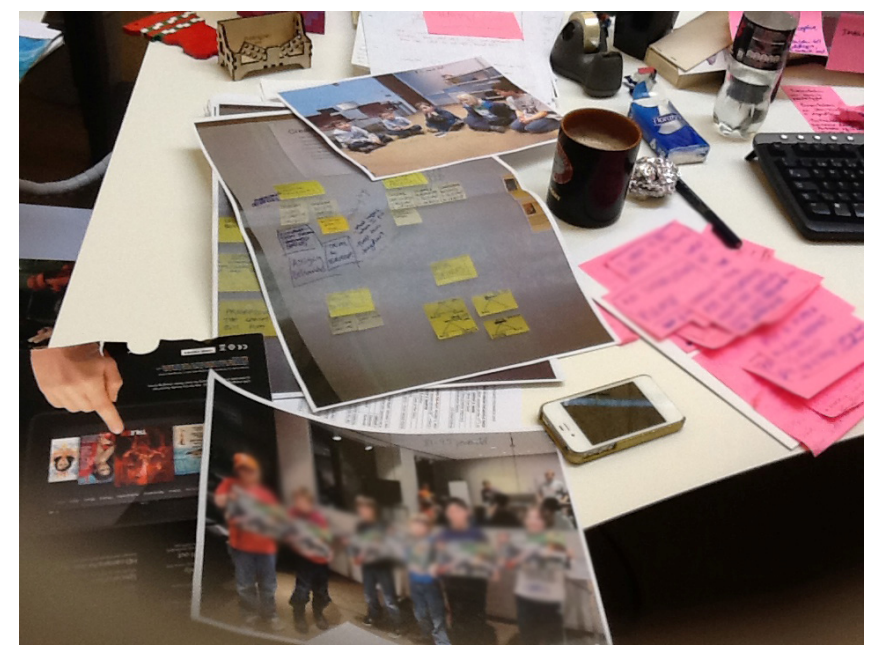

Fig. 1. Typical desktop of a member of the Insights and Early Innovation stream at LEGO Future Lab. Note the stacked post-its on the right side; and in the middle, to the left of the coffee mug, printed out snapshots of a billboard, with the stream member's hand-written notes added.

In general, observations and interviews at the Billund office showed that all employees used post-its for note taking, both privately and at meetings. Many working desks were almost completely covered by post-its, and some employees even had written post-it notes stacked in layers (see Figure 1). Post-its are also used to present ideas for and discuss ideas with others, using the movable billboards as space for sticking - and later moving around with - the post-its. One employee from Insights and Early Innovation described how she would write notes on post-its, because it made the transition from her private work sphere to the sphere of discussing with others easier: she could simply bring the post-its with her personal notes and stick them directly on the billboard at which the discussion was taking place. Another employee from Launch expressed that the small, portable format of the post-it block made it easy to bring to a meeting. It is also the interpretation of the authors of this paper that the sizes of the post-its force the employees to think in a very concise 'to-the-point' format. For visual ideas, drawings on larger paper are also used.

Recently, the team has started utilizing an app called 'Prototype on Paper' for rapid paper prototyping when discussing possible ideas for the design of an app. The basic idea of this tool is that it allows you to sketch the different screens of the app on paper, take snapshots of them with a smart device, and then quickly define links between the different screens by simple finger gestures. It is the impression of the authors that while this is still very new to the employees, they have welcomed the intuitive interface and the spontaneity supported by the system, which hence could prove to be a valued tool further on. 


\subsection{Tools for Collaboration}

Collaborative work within LEGO® Future Lab as a whole (international divisions included) includes both digital and analog tools.

The Billund division of LEGO Future Lab collaborates externally with their counterpart in Los Angeles. These collaborations are conducted via e-mail, infrequent videoconferences and phone calls (due to the time difference), but also via shipping of e.g. physical models from one country to another. Otherwise, the majority of the employees work together within the context of the office. All of the interviewees agreed that they preferred face-to-face contact to electronic communication. In other words, if the employees need input from their colleagues, they usually shout or walk over to the colleague's desk. The employees who do not want to be disturbed, work with their headphones on. Of course, whether the team members go for analog or digital means of collaboration is also dependent on the specific context:

"A prioritized list of the tools I use would be: e-mail, face-to-face, phone/Skype. Actually, I [would prefer not to use] e-mails, but the primary reason for me using them is that we spend much time separated from each other. A lot of our communication happens at nighttime and across distances. It is really a poor form of communication since A) we receive loads of e-mails, hence people tend to not read every single one of them, B) communication is often 'lost in translation'. [...] In general, it is just more beneficial to handle things face-to-face." Michael, Project Leader of new products, Launch.

"I could work from home everyday. It is actually a possibility for me, but I decide not to. Something just happens in that informal communication you have with your colleagues on an everyday basis." Maiken, Digital Product Designer, Pipeline.

"I like meeting face-to-face when communicating with the designers. They prefer to express themselves visually and physically so it just enables communication. Of course they could also just add it to a PowerPoint and send it to me, but it is just easier to do it face-to-face." Louise, Project Leader, Pipeline.

\subsection{Communication with External Collaborators}

Communication in itself, especially with collaborators outside the Billund office, provides a lot of specific problems, many of which stem directly from the geographic dislocation of the collaborators:

"The downside to video communication is that we all need to be online at the same time and that is usually not possible in our case. Another disadvantage is that you do not have the communication in writing as a reference afterwards." Michael, Project Leader of new products, Launch.

Some problems have to do with the same need for face-to-face interaction as described in section 5.2:

"We [the Billund office and external partners in a different time zone] do not communicate on a daily basis and try not to be dependent on each other because communication can be tiresome. It's a bad match time zone wise. In general, just the fact that you are not located the same place is really problematic since you miss out 
on the informal interaction with your colleagues that occurs during the day and that is of great importance. [...]

"[On video communication:] It definitely comes with a barrier. Things are more easily misunderstood or perceived 'stringently' via video. Reading body language is not as easily possible in video communication. The sound is not stable, and delays in connection are also common. It's just not the same as being situated together, and it never will be." Maiken, Digital Product Designer, Pipeline.

"We try to meet up with our Los Angeles team at least once a month to ensure that we are on the same track, but it is a long trip." Louise, Project Leader, Pipeline.

Another issue with respect to the choice of digital vs. analog tools for collaborative work is language barriers between collaborators of different nationalities.

"The language barrier is equally big in writing and video communication, however in some cases people have more time to formulate their wording in writing and hence e-mails come across as a more clear tool for communication. In other cases, video is better since you can better explain your opinion." Michael, Project Leader of new products, Launch.

For collaborators actively working on visual or tangible designs, communicating ideas for such designs over a physical distance also provides special problems:

"We use a lot of e-mails, because a lot of the stuff we see is visuals, drawings, images, and so on. So we usually use attachments in e-mails." Sidd, Creative Lead, Launch.

"It is difficult to convey physical objects via video communication. Sometimes we show the model in front of the camera, but when the receiver does not have an opportunity to feel and touch the model, it is complicated. Other times we build the models in pairs and ship one off to the receiver." Maiken, Digital Product Designer, Pipeline.

The preference for sharing physical models with external collaborators is something that will be dealt with further in section 5.5 below.

\subsection{Tools for Presentation}

With regular intervals, one or more members of LEGO® Future Lab have to present their work either to members from a different stream of LEGO Future Lab, to external partners or to their superiors. Again, the choice of presentation tools depends on the context. For demonstrating e.g. a particular process involving a physical product to a remote collaborator in a different time zone, video logs are often used:

"Recently we have started to use video logs to convey our creative thoughts to our external companies. We send one once a week to keep each other updated." Michael, Project Leader of new products, Launch.

For internal presentations within the specific office, or with external collaborators via video link, physical foam boards and models may be employed in a "show and tell"-like manner, sometimes taking an individual team member's workspace as the starting point. For many purposes, however, PowerPoint seems to be a preferred tool:

"PowerPoint is the one software that everybody uses. It is stupid really, because it is used for tasks that it is not designed for. People use it to do visual work. The reason 
for that is that everyone has access to it. As a designer, I would prefer to use Illustrator to do visual design, but the project managers do not have access to it." Maiken, Digital Product Designer, Pipeline.

Other interviewees have different reasons for seeking supplements to PowerPoint:

"[on plans for new office interior decor:] we want to have more digital screens for several reasons. We would like to have more dynamic content such as videos and loops with info. From a hypothetical standpoint, we hope it will be used more frequently. Then I can take my PowerPoint or Excel sheet, that I already use, and 'shoot' it onto the shared screen for presentations." Michael, Project Leader of new products, Launch.

\subsection{Working with Physical Models}

As much as two-dimensional images are an integral part of the workflow, the interviewees at LEGO ${ }^{\circledR}$ Future Lab all expressed a need for tangible models as part of the displays for discussion of which the billboards are the center. Consequently, practically all billboards are supplemented with tangible models directly in front of them on small desks. Tangible models can include both LEGO prototypes and competitor products. The latter are sometimes supplemented with $\mathrm{QR}$ codes that make it possible to quickly access web links related to these products.

"We display competitor products in the office space. We buy a lot and make an effort to have them physically represented. It is not enough to look at things online when you can touch, feel and play with them instead, try them out and see how they work." Maiken, Digital Product Designer, Pipeline.

"I prefer to work with physical models. If I use LDD [= Lego Digital Designer, see below] it is only to replicate something I already built physically. I always have bricks ready at hand at my desk, just to play around with." Maiken, Digital Product Designer, Pipeline.

LEGO bricks are, as mentioned earlier, also a tool used by the employees in their daily workflow, both for prototyping and for playing during pauses (or, as an employee from Insights and Early Innovation confided, during telephone meetings.) Even though the designers have access to the software LEGO Digital Designer (LDD) that allows the user to build any LEGO model in a virtual environment, brick by brick (and easily generate assembly instructions), none of the interviewed designers preferred working in LDD before the stage of finalizing an idea for presentation, or a final product. In fact, when sharing early prototypes with foreign partners, the Billund office prefers to build an extra version of the prototype and send this to the foreign office, rather than sending a digital version constructed in LDD.

"When we travel we often have boxes of bricks shipped to our destination. People need to work with tangibles. [...]

[On sharing models:] We do not use digital software [LDD]. It takes extra time to do it, you have to do it step by step and construct a building instruction. It is complex, and takes a lot longer than just building an extra copy of the model." Maiken, Digital Product Designer, Pipeline. 
"I use a lot of physical models as you can see on my desk. I can have 6-7 versions of the same model standing while I try to figure out how it should be. [...]

When the models are done, I make them in 3D so it is there and people can always use that file to build again. It is fairly easy to build a model brick by brick. [...]

[Responding to the question of why he does not work from scratch in the digital environment:] It is easier for me to build it [physically], and to change things around, and a lot of the design happens by looking at the pieces - in the digital one you need to go through menus to see them. When you see all the bricks in real life, you are inspired to use certain bricks. That does not happen digitally. Also a lot of troubleshooting happens when you do it physically, in digital it is not as easy as with your hands." Sidd, Creative Lead, Launch.

The need for tangible, physical models in the creative process is evident by the fact that even environments that are intended to be only virtual, e.g. environments visualized as if built in LEGO in one of LEGO's computer games, are sometimes created as full physical models:

"[Explaining the idea behind a large landscape model on the floor:] It is a 'blueprint' model from one of the workshops with our external company, where we tried to make decisions on sizes and dimensions in a digital game. It is a way of making it tangible so instead of spending time on discussing these matters, discussions that could have an academic approach, we just build it instead. Then everybody understands across professional backgrounds and language barriers. We feel that it is easier, and it saves time and development loops." Michael, Project Leader of new products, Launch.

\section{Conclusion}

In general, the case study of LEGO® Future Lab shows that even in a working unit where the use of digital tools is important and mandatory due to a rigidly organized workflow involving many, often geographically dislocated people, individual team members are still dependent on, and prefer using analog tools throughout the larger part of the creative process.

One explanation is the individual's preference for working with something tactile. This goes for post-it notes and pencils as well as working with LEGO bricks. Another explanation is the need to have different objects readily 'at hand', so as to be more easily inspired by the objects (e.g. LEGO bricks, models, images etc.) than one would do when having to look in digital folders for information. In relation to collaboration, the preference for analog communication, in the sense of face-to-face meetings indicates the failure of existing digital communication tools to sufficiently reduce the distance between the communicating partners: Even video-based conversations lack the possibility of direct or even simulated eye contact. Finally, an important factor in the choice of tools is the dependence of multiple types of artifacts in the workspace, i.e. artifacts for presentation, note-taking and physical modeling, items for inspiration etc., and the absence of one overarching digital system to bind all these artifacts together. Rather than using many digital tools for different parts of the process, the 
user typically limits her to a few digital tools (e.g. e-mail, PowerPoint) and takes care of other parts of the process via analog tools.

Especially two aspects in connection with the suggested explanations above have direct implications for the development of digital tools for creative collaboration, such as the ones, which are indeed the focus of the IdeaGarden research project:

1) The need to bridge the gap between the dependence on digital tools and the creative worker's preference for analog ones point towards the integration of tangible tools in the digital environment, e.g. digital pens for use on an interactive whiteboard, or the ability to quickly scan or otherwise identify physical objects and paper notes for use in a collaborative digital environment

2) The LEGO Future Lab team member's dependence on multiple types of artifacts in the daily workflow suggests that a new digital system for creative collaboration either should be simple enough to easily interact or integrate with existing tools in the workplace, or have to provide team members with a myriad of functions, preferably with tangible counterparts, that cover the same bases as their existing tools.

A third aspect, which is of particular importance for the work processes of LEGO Future Lab, but might not be a general problem for creative professionals, is the wish to remove distance between digital collaborators, or to put it differently, bring the online communication situation closer to resembling a physical meeting between the communicating parties.

In the future work of the researchers within the IdeaGarden project, the two aforementioned aspects play a central role in the development of prototypes and software that can facilitate creative collaborative processes. The aforementioned aspect related to removing distance, will, however, not be addressed to the same extent, given that this aspect has less importance in relation to the two other test beds involved in IdeaGarden. [Footnote: For more information on future developments within this research project, please visit www.idea-garden.org]

\section{References}

1. Sellen, A., Harper, R.: Paper as an Analytic Resource for the Design of New Technologies. In: Proceedings of the ACM SIGCHI Conference on Human Factors in Computing Systems, pp. 319-326. ACM (1997)

2. Gaver, W.W.: Situating Action II: Affordances for Interaction: The Social is Material for Design. Ecological Psychology 8, 111-129 (1996)

3. Brereton, M., McGarry, B.: An Observational Study of How Objects Support Engineering Design Thinking and Communication: Implications for the Design of Tangible Media. In: Proceedings of the SIGCHI Conference on Human Factors in Computing Systems, pp. 217-224. ACM (2000)

4. Geyer, F., Reiterer, H.: A Cross-Device Spatial Workspace Supporting Artifact-Mediated Collaboration in Interaction Design. In: Proceedings of the 28th International Conference Extended Abstracts on Human Factors in Computing Systems, pp. 3787-3792. ACM (2010)

5. Schön, D.: The Reflective Practitioner: How Professionals Think in Action. Basic Books, New York (1983) 
6. Fish, J.: Cognitive catalysis: Sketches for a Time-Lagged Brain. In: Goldschmidt, G., Porter, W. (eds.) Design Representation, pp. 151-185. Springer, London (2004)

7. Dorta, T., Pérez, E., Lesage, A.: The Ideation Gap: Hybrid Tools, Design Flow and Practice. Design Studies 29, 121-141 (2008)

8. Jacucci, G., Wagner, I.: Performative Roles of Materiality for Collective Creativity. In: Proceedings of the 6th ACM SIGCHI Conference on Creativity \& Cognition, pp. 73-82. ACM (2007)

9. Christensen, B.T., Schunn, C.D.: The Relationship of Analogical Distance to Analogical Function and Pre-Inventive Structure: The Case of Engineering Design. Memory and Cognition 35, 29-38 (2007)

10. Buur, J., Ylirisku, S.: Designing with Video: Focusing the User-Centred Design Process. Springer, London (2007)

11. Pink, S.: Doing Visual Ethnography, 2nd edn. Sage, Thousand Oaks (2007)

12. Yin, R.K.: Case Study Research - Design and Methods, 4th edn. Sage, Thousand Oaks (2009)

13. Kumar, R.: Research Methodology: A Step-by-Step Guide for Beginners. Sage, London (1999) 